\title{
VINCENT CHALLET
}

\section{PEUPLE ET ÉLITES}

Stratégies sociales et manipulations politiques

dans les révoltes paysannes (France, $\mathrm{XIV}^{\mathrm{e}}-\mathrm{XV}^{\mathrm{e}}$ siècle)

"La campagne? Qui la sait avant le XIV ${ }^{e}$ siècle? Ce grand monde de ténèbres, ces masses innombrables, ignorées [...]. Je n'étais pas en garde, ne m'attendais à rien, quand la figure du Jacques, dressée sur le sillon, me barra le chemin; figure monstrueuse et terrible «' ${ }^{1}$. Peu d'historiens reprendraient aujourd'hui à leur compte ces propos liminaires de Jules Michelet dans le tome qu'il consacra à l'histoire du Moyen Âge et rares sont ceux qui pensent encore que les révoltes paysannes que connut la France au cours de la fin du Moyen Âge furent l'œuvre de masses désorganisées se livrant sans vergogne à leurs plus bas instincts. Cette image est pourtant peu ou prou celle que nous ont léguée les chroniqueurs médiévaux qui ont généralement pour principe d'assimiler tout révolté, qu'il soit paysan ou urbain, à un individu livré à un instinct diabolique et exclu de ce fait de la chrétienté. Le récit des atrocités commises par les jacques au cours de leur insurrection les place clairement en-dehors des normes sociales et comportementales communément admises par la société médiévale. C'est notamment ce que souligne Jean Froissart dans ses "Chroniques«: ils tuèrent un chevalier et le boutèrent en un hastier, et tournèrent au feu et le rostirent, voiant le dame et ses enfans. Apriès ce que dix ou douze eurent la dame efforcie et violé, il les en vorrent faire mengier par force, et puis les fisent morir de male mort ${ }^{2}$. Si le viol fait partie d'une criminalité tant ordinaire qu'irrémissible, celui commis par des rustres sur la personne d'une dame noble marginalise ses auteurs en les situant au-delà des limites de l'acceptable; a fortiori, les accusations rituelles et récurrentes d'anthropophagie placent-elles les révoltés en-dehors du monde chrétien. Ces dernières ne concernent pas, loin de là, les seuls révoltés ruraux: les émeutiers qui, à Montpellier en 1379, ont mis à mort les officiers du duc d'Anjou sont pareillement accusés dans les lettres d'abolition délivrées aux habitants d'avoir dévoré de la

\footnotetext{
${ }^{1}$ Jules MiChelet, Le Moyen Âge, Paris 1981, p. 27.

${ }^{2}$ Les Chroniques de Froissart, éd. par Siméon LUCÉ, vol. V, Paris 1874, p. 101. Pour une analyse détaillée de ce récit, voir Marie-Thérèse DE MEDEIROS, Jacques et chroniqueurs: une étude comparée de récits contemporains relatant la jacquerie de 1358, Paris 1979, p. 45-67.
} 
chair baptisée, à l'instar de bêtes sauvages ${ }^{3}$. Et force est de constater que la chancellerie royale elle-même reprend cette idée d'une inspiration diabolique que l'on retrouve sous la plume des chroniqueurs: les lettres de rémission dénoncent les Toulousains révoltés contre le comte d'Armagnac en 1357 instinctu diabolico inflammati ${ }^{4}$, les Montpelliérains ont agi more Luciferi ${ }^{5}$ et, selon l'auteur de la »Chronique du Religieux de Saint-Denis«, les Tuchins auvergnats et languedociens se sont rebellés diabolico instinctu ${ }^{6}$. Les clercs de la chancellerie royale rejoignent ici les chroniqueurs pour renvoyer les émeutiers dans le camp du Diable alors même qu'ils ne voient pas »le diable partout, ni à tout moment ${ }^{7}$. Ils n'en opèrent pas moins une abusive simplification des insurrections paysannes et se livrent à un évident travestissement de la réalité en masquant l'active participation des élites tant urbaines que rurales à ces mouvements.

La plupart des historiens s'accordent en effet aujourd'hui à penser que les révoltes paysannes de la fin du Moyen Âge, loin d'être de simples flambées de violence imputables à une hypothétique lutte des classes, s'appuient avant tout sur des réseaux de sociabilité dont la force réside en partie sur la constitution de solidarités verticales. Les principales séditions que connut le royaume de France aux $X V^{e}$ et $X V^{e}$ siècles - jacquerie des paysans d'l̂le-de-France et du Beauvaisis de 1357 et Tuchinat languedocien de 1381 à 1384 - de même que leurs avatars à coloration patriotique - Tuchinat auvergnat à partir des années 1360, insurrections paysannes dans la Normandie anglaise et notamment celle du pays de Caux en 1436, Brigands du Forez dont l'activité fut endémique entre 1422 et 1431 - ne doivent leur existence, leur force et parfois leur durée qu'à la quasi-unanimité des communautés villageoises engagées dans la rébellion. La révolte paysanne est d'abord un engagement communautaire avant d'être un choix individuel et Jean le Bel qualifie d'ailleurs à plusieurs reprises

${ }^{3}$ Archives nationales, JJ 119, $\mathrm{n}^{\circ}$ 185, fol. 121-123v; Alexandre GERMAIN (dir.), Histoire de la commune de Montpellier, vol. II, Montpellier 1851, p. 389: Imo, quod maxime exemplo grave est, et abominabile in natura, et alias inauditum, interfectorum corpora aperiebant mucrone, et baptizatas carnes, ut ferae bestiae comedebant. Sur cette émeute, voir Alexandre GERMAN, Une émeute populaire sous Charles V, dans: Académie des sciences et lettres de Montpellier (1847), p. 39-66.

${ }^{4}$ Claude DE VIC, Joseph VAISSÈTE, Histoire Générale de Languedoc, vol. X, Toulouse 1885-1887, preuves, col. 1129-1131 (désormais abrégé en HGL). Il s'agit de lettres de rémission délivrées par le comte d'Armagnac. Sur cette révolte dite »du capage«, voir Jean REGNÉ, La levée du capage et l'émeute toulousaine du 9 mai 1357, dans: Annales du Midi 30 (1917-1918), p. 421-428.

${ }^{5}$ Archives nationales, JJ $119, \mathrm{n}^{\circ} 185$, fol. $121-123 \mathrm{v}$.

${ }^{6}$ Chronique du Religieux de Saint-Denys contenant le règne de Charles VI de 1380 à 1422, vol. I, éd. par Louis-François BELLAGUET, Paris 1842, p. 306 (désormais abrégé en RSD).

${ }^{7}$ Bernard GUENÉE, L'opinion publique à la fin du Moyen Âge d'après la »Chronique de Charles VI du Religieux de Saint-Denis«, Paris 2002, p. 107. 
les jacques de "communautés $\aleph^{8}$ ou de communes. Cette prétention des rebelles à se qualifier de communes et à en revendiquer le titre implique une dimension englobante dont il est possible de se demander si, à l'instar du mouvement anglais de 1381, elle ne doit pas être comprise à l'échelle du royaume et être assimilée à une communitas regni $i^{9}$. Cette connexion entre révoltes paysannes et politique générale du royaume est sans doute l'une des grandes nouveautés des $X \Gamma V^{e}$ et $X^{e}$ siècles et les contacts noués entre Guillaume Carle et le prévôt des marchands de Paris, Étienne Marcel, à l'occasion de la jacquerie en sont un premier indice.

Sans doute faut-il en effet reconsidérer le lien entretenu au cours de leurs révoltes par les paysans avec la politique suivant en cela l'intuition déjà ancienne d'Eric Hobsbawm qui définissait la politique en contexte paysan comme wla relation des paysans à d'autres groupes sociaux [...] et à des institutions plus larges, telles que le gouvernement ou l'État national ${ }^{10}$ et insistait sur la nécessité pour les groupes paysans d'assurer la relation entre microcosme et macrocosme compte tenu de leur incapacité à s'imposer par euxmêmes sur la scène politique. Ses idées furent reprises et appliquées pour l'époque médiévale par Rodney Hilton puis, plus récemment, par Paul Freedman qui démontra l'aptitude des communautés paysannes à réutiliser à leur profit des concepts politiques complexes forgés en-dehors de leur propre univers ${ }^{11}$. Il convient d'ailleurs de ne pas perdre de vue que la rébellion n'est jamais que l'une des possibilités offertes aux communautés paysannes pour entrer dans le champ politique et qu'elle doit être replacée au sein d'un long processus susceptible de faire intervenir la formulation de doléances ou l'appel à la justice royale. Les révoltes paysannes ont jusqu'à présent été considérées par les historiens au travers du prisme de trois principaux modèles théoriques, dont le premier et le plus connu n'est autre que le modèle d'explication marxiste qui envisage la rébellion comme un conflit de classes entre tenanciers et propriétaires du sol ou entre contribuables et pouvoir seigneurial ou royal. Cette théorie, fondée sur l'existence de fortes tensions sociales au sein de l'Europe médiévale, peine cependant à expliquer la constitution d'une conscience de classe au sein de la société paysanne à l'échelle d'un royaume et néglige la présence de liens de solidarité verticale entre les plus désargentés des manouvriers et les élites ainsi que la violence des conflits locaux internes à chaque communauté. Une deuxième tentative de modélisation consiste à cher-

\footnotetext{
${ }^{8}$ Chronique de Jean le Bel, vol. I, éd. par Jules Viard, Paris 1905, p. 257: le meschief fut si multiplié que les communaultés eussent tous les nobles destruit.

9 John WatTs, The Pressure of the Public on Later Medieval Politics, dans: Linda CLARK, Christine CARPENTER (dir.), Political Culture in Late Medieval Britain 2004 (The Fifteenth Century, 4), p. 159-180.

${ }^{10}$ Eric HobSBawm, Peasants and Politics, dans: Journal of Peasant Studies 1/1 (1973), p. 3-22.

${ }^{11}$ Paul FreedMAN, Images of the Medieval Peasant, Stanford 1999.
} 
cher la clef des rébellions paysannes dans l'analyse de conditions politiques locales: la rébellion deviendrait alors un choix rationnel fait par des individus et serait la conséquence logique d'une situation donnée. Selon cette théorie, la révolte éclaterait lorsque les paysans estimeraient que les profits qu'ils peuvent en tirer sont supérieurs aux risques qu'ils encourent. Mais c'est faire bon marché de facteurs irrationnels tels que les motifs religieux et ne laisser aucune place aux émotions et à leur transmission, dont le rôle est pourtant si considérable dans maintes émeutes ${ }^{12}$. Ce dernier élément est mieux pris en considération par la théorie prophétique qui étudie la manière dont des mouvements religieux à forte dimension eschatologique ont pu se transformer en rébellions ${ }^{13}$. Cette façon de voir, si elle reconnaît l'importance de la prédication et du charisme personnel, ne peut toutefois s'appliquer qu'à des mouvements très particuliers tels que la rébellion hussite ou, pour le royaume de France, la croisade des Pastoureaux et ne saurait être retenue pour des rébellions dépourvues de toute composante religieuse. Il n'en reste pas moins que l'ensemble de ces théories néglige un élément fondamental qui sous-tend la plupart des révoltes rurales de l'époque médiévale, à savoir le développement d'une culture politique commune partagée sinon, à l'échelle d'un royaume, du moins à celle d'une province. Cette culture politique consiste en une vision populaire de la justice, un recours habituel à des actions collectives - qu'elles soient violentes ou non -, un type spécifique de relations vis-à-vis des seigneurs ou des agents royaux, la pratique d'actions judiciaires et d'appels aux tribunaux royaux, une histoire et une mémoire commune ainsi qu'une conception identique du roi et du gouvernement. Dans le contexte du Bas Moyen Âge et de la guerre de Cent Ans, cette culture politique des communautés villageoises s'articule essentiellement autour des notions de solidarité face à des menaces extérieures et d'autodéfense.

Ces valeurs d'autodéfense sont notamment révélées à l'occasion de la jacquerie. Guillaume Carle s'impose certes partiellement comme chef du mouvement mais il a sous ses ordres nombre de capitaines, chaque paroisse ralliée au mouvement ayant envoyé vers les points de ralliement ses hommes dotés de leur propre chef et de bannières parfois fleurdelisées ${ }^{14}$. Mouvement d'auto-

\footnotetext{
${ }^{12}$ Ainsi de la psychologie des foules, de l'environnement sonore (cris et cloches) et de son impact, du déploiement de signaux visuels tels que les bannières ou du charisme d'un leader particulier. Voir Vincent CHALLET, Émouvoir le prince. Révoltes populaires et recours au roi en Languedoc vers 1380, dans: Hypothèses (2001), p. 325-333.

${ }^{13}$ Norman COHN, Les fanatiques de l'Apocalypse: millénaristes révolutionnaires et anarchistes mystiques au Moyen Âge, Paris 1983.

${ }^{14}$ Siméon LUCÉ, Histoire de la Jacquerie, Paris 1895. Sur la jacquerie, on consultera aussi G. FOURQUIN, Les campagnes de la région parisienne à la fin du Moyen Âge du milieu du XIII $^{c}$ siècle au début du XIV ${ }^{c}$ siècle, Paris 1964 et ID., Les soulèvements populaires au Moyen Âge, Paris 1972. On y ajoutera J. FLAMMERMONT, La Jacquerie en Beauvaisis, dans: Revue historique 19 (1879), p. 123-143 et R. CAZELLES, La Jacquerie fut-elle un mouve-
} 
défense devant les pillages des compagnies anglaises, la jacquerie naît d'une exaspération face à ces ravages, portée à son paroxysme par la trahison des nobles. Que le ralliement des paysans révoltés se fasse au son du tocsin, de même que la présence de prêtres à la tête des troupes démontre toute l'importance du cadre paroissial. L'analyse du Tuchinat languedocien qui sévit de 1381 à 1384 va dans le même sens: les Tuchins ne sont pas des »bandes d'asociaux affamés et sans autre programme que de survivre aux dépens de l'ordre établi ${ }^{15}$ mais possèdent une organisation qui repose sur des liens de sociabilité et de solidarité villageoise. Regroupés en bandes d'une vingtaine ou d'une trentaine de membres qui prêtent un serment d'obéissance à un capitaine librement choisi, les Tuchins arborent leurs propres bannières et disposent même d'une fanfare. En outre, leur recrutement se fait dans un rayon d'une trentaine de kilomètres tout au plus et en fonction d'une origine géographique précise: ainsi, à Bagnols-sur-Cèze où se rejoignent en 1382 quatre groupes différents, le premier compte dans ses rangs des hommes venus des villages de l'ouest de Bagnols, le second comprend des villageois de l'est de cette ville tandis que les membres des deux derniers proviennent de l'espace situé entre Bagnols et Uzès. La cohérence de ces bandes tient donc au fait que les rebelles sont d'abord des voisins, ce qu'indique aussi le terme de compagnons qu'ils se donnent ${ }^{16}$. Les révoltes de la fin du Moyen Âge naissent le plus souvent d'un sentiment collectif de danger face aux agressions que constituent les pillages des hommes d'armes; menacé dans sa survie et dans son ensemble, le village se doit alors d'apporter une réponse, sinon unanime du moins communautaire. Si la courte durée de la jacquerie ne permet guère d'envisager l'insertion des rebelles au sein d'un réseau villageois tout en révélant l'enracinement dans un cadre paroissial ${ }^{17}$, on constate en revanche que les Tuchins, loin d'être en permanence sur le pied de guerre, ne se rassemblent qu'à l'occasion d'opérations militaires ciblées et vivent le reste du temps au sein de leur communauté, occupés à divers travaux agricoles tels que les moissons ou les vendanges. S'il est impossible de mettre en évidence un système identique à celui de la rre-

ment paysan?, dans: Comptes rendus de l'Académie des inscriptions et belles lettres (1978), p. $654-666$.

is Michel Mollat, Philippe WolfF, Ongles bleus, Jacques et Ciompi. Les révolutions populaires en Europe aux XIV et XVt siècles, Paris 1970, p. 185.

${ }^{16}$ Sur le Tuchinat, que l'on me permette de renvoyer à mes propres travaux: Vincent CHALLET, Mundare et auferre malas erbas: la révolte des Tuchins en Languedoc, thèse de doctorat, dactyl., université Paris I, 2002.

17 Cette différence essentielle entre un Nord de la France où les solidarités villageoises s'expriment surtout dans un cadre paroissial et un Midi où se remarque une slaïcisation ‘ progressive des solidarités avait déjà été soulignée par Monique BOURN, Robert DURAND, Vivre au village au Moyen Âge. Les solidarités paysannes du XI' au XII' siècles, Rennes 2000. 
lève ‘ instituée par les paysans allemands en $1525^{18}$, la célébration en commun des rites traditionnels de la sociabilité villageoise démontre à l'envi que la rébellion est vécue à l'échelle communautaire bien plus qu'individuelle. L'un des capitaines tuchins célèbre son mariage dans l'église paroissiale avant d'offrir à ses invités un repas de noces au cours duquel sont consommés du vin et des victuailles saisis au cours de la révolte. En une autre occasion, les Tuchins convient les villageois à un méchoui où sont rôtis, avec la bénédiction de leurs propriétaires, des agneaux récupérés sur des gens d'armes. La révolte se nourrit ainsi du boire et du manger en commun et révèle sa dimension communautaire. Enfin, ce que l'on sait du brigandage normand pendant l'occupation de la province par les Anglais correspond également à ce type de comportement: regroupés en bandes mobiles et liés entre eux par un serment, les Brigands ou partisans normands ont des éclaireurs, des guides qui continuent de résider dans les villages et leur expédient des recrues. À l'instar des Tuchins languedociens qui observent un strict partage égalitaire du butin, les Brigands tiennent au sein de chaque groupe une comptabilité qui assure à chacun sa part des prises de guerre. Ils possèdent aussi des relais dans la population et peuvent compter sur le soutien de prêtres ruraux. L'une de ces compagnies s'est même dotée d'un parc à bétail, d'un dépôt de fourrage, de réserves de nourritures et de boissons, va vendanger pour son compte les vignes d'un curé et fait du vin en forêt avec des pressoirs installés sur place ${ }^{19}$. En outre, bien des paysans se font les complices de ces Brigands en assurant la transmission des nouvelles, la vente sur le marché de leur butin ou, plus simplement, en les ravitaillant ${ }^{20}$.

La composition sociale des révoltés n'infirme en rien, lorsqu'il est possible de la connaître, cette constatation et ces mouvements bénéficient d'un engagement notoire non seulement d'une large partie de ce qu'il est convenu désormais d'appeler des »élites villageoises« mais aussi d'éléments venus du bas clergé, de la noblesse rurale ou d'hommes de loi. Ce phénomène particulièrement bien connu en ce qui concerne les révoltes urbaines - que l'on songe un instant au statut social d'un Salvestro de Médicis impliqué dans la révolte des Ciompi florentins, d'un Étienne Marcel à Paris ou d'un Jacques Van Artevelde à la tête des troupes gantoises - est tout aussi vrai à propos des mouvements ruraux et les indices de cette prise en charge des revendications paysannes par

${ }^{18}$ Ce système permettait à chaque village rallié d'envoyer un homme sur quatre pendant huit jours et d'instaurer ainsi un système de roulement; voir Georges BISCHOFF, Aspects militaires de la guerre des paysans en Alsace, dans: Violence et contestation au Moyen Âge, Actes du $114^{e}$ congrès national des Sociétés savantes, Paris 1990, p. 249-266.

${ }^{19}$ Germain LEFEVRE-PONTALIS, Épisodes de l'invasion anglaise: la guerre de partisans dans

la Haute-Normandie (1424-1429), dans: Bibliothèque de l'École des chartes 54 (1893), p. $475-521$.

${ }^{20}$ Roger JOUET, La Résistance à l'occupation anglaise en Basse-Normandie (1418-1450), Caen 1969 (Cahier des Annales de Normandie, 5). 
une élite sociale sont si nombreux que les explications de type individuel ne résistent guère à l'analyse. La participation d'élites rurales aux mouvements paysans avait déjà été démontrée par Rodney Hilton à propos de la révolte des travailleurs anglais en 1381. S'appuyant sur les actes d'accusation et les registres de confiscation dressés par la monarchie anglaise à l'issue des troubles, il notait que si la paysannerie constituait la grande majorité des individus poursuivis, les artisans ruraux et les représentants des métiers de l'alimentation charpentiers, maçons, tailleurs, boulangers, bouchers, etc. - étaient loin d'être absents et, qu'au sein même de la paysannerie, un nombre notable d'insurgés étaient des tenanciers aisés dont les terres avoisinaient ou dépassaient cinquante acres. Rodney Hilton en concluait qu'il "s'agissait de la révolte de tous les gens se situant en dessous de ceux qui étaient seigneurs dans les campagnes ${ }^{21}$. Le royaume de France ne possède malheureusement pas de documents permettant d'établir avec autant de précision un tableau statistique de la composition sociale des révoltés et il faut en la matière se contenter de données éparses, notamment, en dépit des problèmes de représentativité qu'elles posent ${ }^{22}$, celles livrées par les lettres de rémission. En l'occurrence, s'il est difficilement contestable que la jacquerie ait été un mouvement paysan et que plusieurs lettres de rémission se terminent par la mention comme il soit homme de labour qui a à cueillir et mettre à sauveté ses biens ${ }^{23}$, les artisans et autres gens de métier occupent au sein de cet ensemble documentaire une place prépondérante. Bouchers, maçons, tonneliers, charrons, forgerons ou marchands de volailles furent des membres actifs de la jacquerie à l'instar de ce Mathieu de Leuvel, maçon de son état, qui fit exécuter en présence de deux ou trois cents personnes un messager du roi de Navarre ${ }^{24}$. La composition sociale $\mathrm{du}$ Tuchinat languedocien paraît relativement identique: à Bagnols-sur-Cèze, aux côtés de nombreux brassiers et famuli, se trouvent deux tailleurs de pierre, deux cordonniers et un forgeron ${ }^{25}$. Les artisans se retrouvent d'ailleurs souvent en position intermédiaire à l'intérieur de la société rurale et constituent une sorte de sclasse moyenne،, ni notables, ni miséreux. À partir de la situation des villages biterrois au début du XIV siècle et notamment de l'exemple de Rou-

\footnotetext{
${ }^{21}$ Rodney HILTON, Les Mouvements paysans du Moyen Âge, Paris 1979, p. 232. L'auteur note aussi que "la proportion des gens aisés par rapport à ceux qui avaient une petite ou médiocre fortune et la proportion des artisans par rapport aux agriculteurs suggèrent que la composition sociale des bandes rebelles était le reflet de la stratification sociale contemporaine«.

${ }_{22} \mathrm{Ne}$ serait-ce que parce que les nobles sont surreprésentés dans le corpus des lettres de rémission. Ils constituent en effet $5,7 \%$ des exposants alors qu'ils ne seraient que de 1 à $3 \%$ de la population du royaume. Sur ces problèmes, voir Claude GAUVARD, De grace especial. Crime, État et société en France à la fin du Moyen Âge, vol. I, Paris 1991 (Publications de la Sorbonne), p. 74.

${ }^{23}$ LUCÉ, Histoire de la Jacquerie (voir n. 14), p. 63.

${ }^{24}$ Exemple cité et commenté par GAUVARD, De grace especial (voir n. 22), vol. I, p. 422.

${ }^{25}$ Challet, Mundare et auferre (voir n. 16), vol. 1, p. 385.
} 
jan où éclate dans les années 1320-1330 une crise interne destinée à obtenir une meilleure justice fiscale et le respect des institutions communales, Monique Bourin s'était déjà demandée si ce n'est pas »dans le groupe artisanal que la conscience et l'engagement politiques sont les plus aigus $\kappa^{26}$. Ainsi, plutôt que de conclure à des mouvements paysans initiés et menés par des gens de métier, je rejoindrai ici les observations de Rodney Hilton: les révoltes rurales entraînent dans leur sillage tous les membres d'une communauté, qu'ils soient ouvriers agricoles, paysans aisés ou artisans. L'étonnant n'est donc pas de rencontrer des éléments de l'artisanat dans les révoltes paysannes, ce serait au contraire leur absence qui serait surprenante.

Les artisans ne furent pas le seul groupe social à s'engager au sein des mouvements paysans et la plupart des insurrections virent la participation du bas clergé, de la petite noblesse ainsi que d'hommes de loi. Le Tuchinat languedocien est ici un très bon exemple de cette implication d'élites non villageoises dans les révoltes paysannes. La quasi-totalité des lettres de rémission accordées pour fait de Tuchinat furent délivrées à des écuyers ou des damoiseaux placés à la tête de compagnies militaires par leurs voisins et dépendants et qui assumèrent le rôle de capitaine sans que cela ait toujours été, comme ils le prétendirent ultérieurement, sous la contrainte. C'est ainsi que des Tuchins vinrent chercher Jean de Ferrières parce qu'il avait l'habitude de servir dans les armées du roi, l'élirent capitaine, reçurent son serment de fidélité et le suivirent dans diverses opérations contre les gens d'armes ${ }^{27}$. Certes, une partie de ces nobles abandonna les révoltés pour se rallier au pouvoir royal et participa même à la répression du mouvement. Quelques-uns pourtant allèrent jusqu'au bout de leur engagement et furent capturés à la tête de leurs troupes avant d'être exécutés: c'est le cas de Bérenger de Montpezat, damoiseau et seigneur de Saint-Geniès-de-Malgoirès ${ }^{28}$, décapité pour crime de lèse-majesté et sans doute de Raymond de Corneilhan, chevalier de Puisserguier ${ }^{29}$. Le plus illustre des nobles ralliés au Tuchinat n'en demeure pas moins ce Pierre de Bré, chevalier, rejeton de cette famille Aubert qui en la personne d'Innocent VI avait fourni un pape à la chrétienté, et devenu à la faveur de son mariage avec Jacqueline de Bruyères détenteur de la terre et baronnie de Capendu dans la sénéchaussée de Carcassonne ${ }^{30}$. Doté de solides relations au sein de la région, il

${ }^{26}$ Monique Bourin-DerruaU, Villages médiévaux en Bas-Languedoc. Genèse d'une sociabilité (X'-XIV siècle), vol. 2, Paris 1987, p. 323.

${ }^{27}$ Archives nationales, JJ 131, $\mathrm{n}^{\circ}$ 226, fol. 146; publ. dans HGL (voir n. 4), vol. X, preuves, col. 1735.

${ }^{28}$ Archives nationales, JJ 126, $\mathrm{n}^{\circ} 117$, fol. 73; Saint-Geniès-de-Malgoirès, canton de SaintChapte, arrondissement de Nîmes, Gard.

29 Archives nationales, JJ 149, $\mathrm{n}^{\circ}$ 1, fol. 1; publ. dans HGL (voir n. 4), vol. X, preuves, col. 1867. Puisserguier, canton de Capestang, arrondissement de Béziers, Hérault.

${ }^{30}$ CHALlET, Mundare et auferre (voir n. 16), vol. 1, p. 212-216. 
parvient à regrouper autour de lui à Aigues-Vives ${ }^{31}$ un nombre important de Tuchins et entretient des relations suivies avec les consuls de Narbonne qui, au même moment, affrontent les troupes de leur vicomte Amaury VII. Pierre de Bré s'est d'ailleurs illustré dès les débuts du Tuchinat en attaquant les bagages du duc de Berry et en pillant ses chariots ce qui ne l'empêche nullement de se trouver à Carcassonne en novembre 1382 pour y ratifier un accord privé en présence de l'évêque de la ville et du sénéchal. Personnage clef du Tuchinat dans la sénéchaussée de Carcassonne, Pierre de Bré agit à une échelle qui dépasse largement celle de sa seigneurie et se voit même affubler par le Religieux de Saint-Denis du qualificatif d'imperator ${ }^{32}$. Il fut néanmoins capturé puis décapité à Carcassonne sans doute au début de l'année 1383 pour crime de lèse-majestés ${ }^{33}$. Si le cas de Pierre de Bré illustre magistralement l'implication de certains nobles dans les mouvements paysans, il témoigne aussi des relations entre insurgés et hommes de lois.

Pierre de Bré fut en effet en relation avec deux juristes de haut vol originaires de Carcassonne, maître Bernard Tissière et Pierre Boyer. Bernard Tissière, qualifié de notaire de la Cité de Carcassonne, était aussi coseigneur d'Aragon et d'Homps en $1371^{34}$ et fut viguier de la terre du monastère de Lagrasse. Or, ce notaire de Carcassonne fut reconnu coupable d'avoir reçu plusieurs fois Pierre de Bré dans sa maison d'Aragon et d'avoir fourni aux Tuchins en diverses occasions des armures et des vivres ${ }^{35}$. Cette sentence ne fut cependant prononcée qu'à titre posthume en 1390 , alors même que de son vivant, nul n'empêcha Bernard Tissière de continuer à exercer l'office de viguier de l'abbaye de Lagrasse et de demeurer coseigneur d'Aragon. Pierre Boyer, docteur ès décrets du Bourg de Carcassonne et héritier de Bernard Tissière pour la seigneurie d'Aragon, joua un rôle capital comme conseiller juridique pour les communautés de Languedoc: il fut à l'origine de plusieurs appels contre le duc

${ }^{31}$ Aigues-Vives, canton de Capendu, arrondissement de Carcassonne, Aude.

${ }^{32}$ RSD (voir n. 6), vol. I, p. 310 . Michel Pintoin l'appelle Pierre de Bruyères et en fait le capitaine général des Tuchins d'Auvergne. Nous avons démontré ailleurs que Pierre de Bré et Pierre de Bruyères n'étaient qu'un seul et même individu; voir CHALLET, Mundare et auferre (voir n. 16), vol. 1, p. 212-216.

${ }^{33}$ HGL (voir n. 4), vol. IX, p. 911 , n. 2, et vol. X, preuves, col. 1798.

${ }^{34}$ Pour la seigneurie d'Aragon, voir Alphonse MAHUL, Cartulaire et archives des communes de l'ancien diocèse et de l'arrondissement administratif de Carcassonne, vol. I, Paris, 18571882, p. 49. Bernard Tissière est mentionné comme coseigneur d'Homps dans le $"$ Livre des dénombrements et aveux des fiefs tenus par les nobles de la sénéchaussée de Carcassonne de l'an 1371 «, dont une copie a été faite par Dom Pacotte et se trouve aux Archives départementales de l'Hérault, A 242, fol. 42: "Maître Bernard Teissiere de la ville de Carcassonne les an et mois dessudits [mars 1371] declare avoir juridiction au lieu de Homps par indivis avec les autres coseigneurs et en pareage avec le roi lequel il tient suivant les coutumes de la vicomté de Paris«. Homps, canton de Lézignan-Corbières, arrondissement de Narbonne, Aude; Aragon, canton d'Alzonne, arrondissement de Carcassonne, Aude.

${ }^{35}$ Archives nationales, JJ 146, $\mathrm{n}^{\circ}$ 223; publ. dans HGL (voir n. 4), vol. X, preuves, col. 1796. 
de Berry, en particulier pour retarder le paiement de divers subsides levés, ce qui fut fait ou grant prejudice $d u$ Roy et $d u$ bien commun ${ }^{36}$ et aurait été l'auteur de lettres diffamatoires, contenans pluseurs injurieuses paroles contre Simon de Cramaud qui était pour lors évêque d'Agen et présidait le conseil de Jean de Berry siégeant en Avignon. Il se serait également impliqué dans la rébellion en conseillant aux diverses communautés de faire genz d'armes et capitainnes. Pierre Boyer était un juriste influent, déjà employé avant la révolte par les consuls de Montpellier puis par ceux du Bourg de Carcassonne qui le désignèrent pour faire partie de l'ambassade chargée d'obtenir de Charles $V$ le rappel du duc d'Anjou et un certain nombre de concessions fiscales ${ }^{37}$. Conseiller juridique écouté des communautés languedociennes, il n'en était pas moins proche du pouvoir royal et se vit délivrer des lettres d'anoblissement le 2 avril $1381^{38}$. En dépit de son action au service des communautés languedociennes et de sa responsabilité dans le Tuchinat, il fit une remarquable carrière dans l'administration royale, devenant régent de la sénéchaussée de Carcassonne en 1389, puis lieutenant du sénéchal jusqu'en 1400. Plusieurs de ses descendants devaient même occuper après sa mort les postes de juge des crimes ou de juge mage de la sénéchaussée de Carcassonne ${ }^{39}$. La carrière et le rétablissement de Pierre Boyer furent tout à fait spectaculaires pour un homme qui n'avait pas hésité à prendre le parti des communautés de Languedoc contre le lieutenant du roi. Lors du voyage de Charles VI qui marqua un changement de cap dans la politique royale vis-à-vis de sa lointaine province, il avait sans doute le profil idéal des hommes que recherchaient les Marmousets pour les placer à la tête des institutions royales: Pierre Boyer avait servi fidèlement le roi et le duc d'Anjou mais en même temps les communautés de la sénéchaussée de Carcassonne pouvaient se sentir proches de lui, puisqu'il avait pris une part active à leur rébellion contre le duc de Berry et qu'il était lié à Bernard Tissière, lui-même complice de Pierre de Bré. En somme, sans avoir été à proprement parler un Tuchin, Pierre Boyer pouvait se targuer d'avoir œuvré pour le »bien public« et celui de ses concitoyens sans jamais avoir réel-

\footnotetext{
${ }^{36}$ Ibid., col. 1798.

${ }^{37}$ Sur cette ambassade, voir Alphonse BLANC, Le rappel du duc d'Anjou et l'ordonnance du 25 avril 1380, dans: Bulletin philologique et historique du comité des travaux historiques et scientifiques (1899), p. 191-212.

${ }^{38}$ Archives nationales, JJ 118, $\mathrm{n}^{\circ} 408$, fol. 217: Nos, accepto ex plurimum testimonio laudabili, dilectum nostrum magistrum Petrum Boierii, decretorum doctorum incolam nostre senescallie Carcassone, premissis graciarum dotibus insignitum atque carissimo domino genitori nostro, cui Christus indulgeat, et nobis in officio consiliarii nostri in curia nostra senescallie Carcassonne diu et fideliter servisse [...] Per Regem, presente domino Andegavense. P. de Manhac.

${ }^{39}$ MAHUL, Cartulaire (voir n. 34), vol. I, p. 274-277. Ainsi Raymond Boyer, juge des crimes et viguier de Carcassonne de 1480 à 1487; Arnaud Boyer, seigneur de Montclar, juge mage de Carcassonne de 1500 à 1525; son fils Pierre Boyer, juge mage de 1534 à 1539 et enfin Jean Boyer juge mage en 1546.
} 
lement trahi son roi. Sa position médiane en faisait donc un interlocuteur privilégié pour un pouvoir royal désireux de rétablir la paix civile en Languedoc. Bernard Tissière et Pierre Boyer ne sont jamais que deux exemples particulièrement marquants parmi d'autres: les liaisons entre Tuchins et hommes de loi furent en effet constantes et sont également attestées dans la sénéchaussée de Beaucaire. Au cours des négociations qui eurent lieu entre les capitaines tuchins et le conseil du duc de Berry, les rebelles se virent remettre des copies de lettres octroyées par la chancellerie ducale, demandèrent quelques jours pour les examiner et finirent par les rejeter en disant qu'il s'agissait de documents accordant une trêve au lieu des lettres d'abolition en bonne et due forme qu'ils avaient réclamées. Un tel comportement surprend de la part de brassiers illettrés et ceci même si l'on est en droit de supposer l'existence chez les paysans languedociens de ce que les historiens anglo-saxons qualifient de practical literacy ou d'insurgent literacy, c'est-à-dire une capacité à reconnaître dans un document écrit le nom de leurs villages ou de leurs terres et une dizaine ou une vingtaine de termes latins qui les rendent aptes à reconnaître les principales articulations d'une charte qui leur est présentée ${ }^{40}$. C'est sans doute ici cette disposition à laquelle il faut ajouter l'examen des modes de scellement ${ }^{41}$ qui alerta les capitaines tuchins et les incita à recourir à une étude plus approfondie du document. Ils procédèrent alors à cette expertise en faisant appel à des juristes ou à des notaires au service des consulats de la région. Les révoltes paysannes ont ainsi pu s'appuyer sur des élites sociales aussi bien internes à la communauté villageoise - tenanciers et laboureurs aisés, artisans et en particulier forgerons - qu'externes - nobles et hommes de loi. Il reste toutefois à comprendre ce qui a pu déterminer ces élites à prendre à un moment donné le chemin de la révolte.

L'une des explications communément admises s'apparente à une déclinaison du thème de la paupérisation de ces élites et cette thèse a notamment été avancée en ce qui concerne les éléments les plus aisés de la paysannerie. $D$ 'après les tenants de cette théorie, les mouvements paysans seraient non des révoltes de la misère mais des réactions violentes et occasionnelles de »coqs de village« menacés par un déclassement social dans une conjoncture économique difficile. Guy Fourquin a ainsi pu noter que la jacquerie avait surtout concerné les parties les plus peuplées d'Île-de-France, celles qui bénéficiaient

\footnotetext{
${ }^{40}$ Ces notions de pratical literacy et d'insurgent literacy ont été explicitées et utilisées par Michael T. ClanCHY, From Memory to Written Record, England 1066-1307, Oxford 1993, et par Steven JUSTICE, Writing and Rebellion: England in 1381, Berkeley 1994.

${ }^{41}$ Les lettres d'abolition réclamées auraient du être scellées du grand sceau de majesté des rois de France et comporter un sceau de cire verte sur lacs de soie rouge et vert, mode de scellement utilisé à la chancellerie royale pour les actes à valeur perpétuelle. Or, les lettres présentées aux Tuchins ne comportaient qu'un sceau de cire rouge qui était probablement celui de la chancellerie du duc de Berry, lieutenant du roi en Languedoc.
} 
des meilleures terres arables et non les secteurs les plus défavorisés ${ }^{42}$. Mais ces régions purement céréalières et totalement dépourvues de vignes étaient précisément celles qui avaient subi de plein fouet la longue stagnation des prix céréaliers alors même que les prix industriels connaissaient une forte progres$\operatorname{sion}^{43}$. Si l'on ajoute à cette »scission des prix« le poids des impôts royaux d'autant plus fort que l'on entre dans la guerre de Cent Ans, la pratique des »prises« et les pillages opérés par les gens d'armes, en 1356, »le point de rupture est atteint tant sur le plan économique que politique et social ${ }^{44}$. La jacquerie serait donc un mouvement de laboureurs et paysans aisés mais en voie de paupérisation en raison d'une crise économique et politique qui aurait comme conséquence de niveler par le bas les fortunes paysannes: ils réagiraient ainsi en tant qu'élites sociales menacées de disparition. A contrario, le calme qui règne dans les campagnes parisiennes entre 1450 et 1530 serait en partie la conséquence d'une reconstruction agricole qui favorise de riches fermiers, lesquels peuvent alors jouer un rôle de modérateur et de régulateur social au sein de leurs villages. Si cette théorie n'est pas à écarter totalement, elle peine néanmoins à expliquer l'active participation d'artisans plutôt favorisés par cette scission des prix et la relative unanimité des communautés paysannes. En Languedoc d'ailleurs, le ressort économique de la crise des Tuchins ne semble guère évident puisque, vers 1380 , le haut niveau des salaires agricoles dont bien des paysans sont dépendants s'avère plutôt favorable aux brassiers et que la stagnation des prix céréaliers contribue à augmenter leur pouvoir d'achat $^{45}$. Même en tenant compte d'une aggravation de la pression fiscale par le biais des impositions royales, il paraît difficile de voir dans une paupérisation accrue des paysans le moteur essentiel de la révolte. Une telle situation est certes défavorable aux gros exploitants qui ont besoin de recourir à une maind'œuvre salariée à l'occasion des grands travaux agricoles mais le Languedoc des années 1380 est plutôt en situation microfundiaire et la culture de la vigne atténue sans doute les effets de cette stagnation. Au surplus, toute hypothèse de type économique doit sans doute se combiner avec une théorie explicative de nature politique.

$\mathrm{Si}$, en effet, l'historien peine souvent à définir des élites rurales par un statut social parfois fluctuant, les élites politiques, elles, se laissent en revanche plus facilement appréhender. À dire vrai, dans la plupart des communautés paysannes, les élites sociales se révèlent aussi être des élites politiques dans le sens

\footnotetext{
${ }^{42}$ FOURQUIN, Les campagnes de la région parisienne (voir n. 14), p. 232-240.

${ }^{43}$ Sur cette scission des prix qui affecte la Normandie tout autant que la région parisienne, voir Guy BoIs, Crise du féodalisme, Paris 1976, p. 83.

${ }^{44}$ Ibid., p. 264.

${ }^{45}$ Faute d'études économiques globales concernant l'ensemble du Languedoc, on se contentera des analyses menées pour le Toulousain par Philippe WOLFF, Commerce et marchands de Toulouse (vers 1350-1450), Paris 1954.
} 
où elles participent, ne serait-ce qu'à un niveau minime, à la gestion du pouvoir communal et à ses relations avec les autorités royales. Dans les villages du Nord du royaume de France où, malgré la présence d'institutions communales définies par les chartes de franchise, les solidarités primordiales continuent de s'exprimer dans un cadre paroissial, c'est souvent la fabrique qui assure, aux côtés du curé, un rôle de direction de la communauté y compris pour des activités extra-religieuses alors qu'elle n'était apparue que comme un instrument de gestion collective de l'église. Les marguilliers placés à la tête de ces fabriques sont, dans les villages dépourvus de commune ou de franchise, les seuls représentants de la communauté et continuent à jouer un rôle essentiel même après l'apparition d'échevins et de jurés désignés au sein des assemblées villageoises. En tout état de cause, marguilliers et jurés se recrutent généralement parmi cette catégorie un peu floue des whommes les plus honorables« de la paroisse ou du village au sein de laquelle se retrouvent les différentes composantes des élites rurales. Or, la jacquerie s'effectue dans un cadre paroissial dont témoignent la présence du clergé et la convocation par le biais du tocsin et mobilise cette même élite sociale qui assume également un rôle directionnel au sein de la communauté.

À cette catégorie des "honorables hommes «, répond dans le Midi, celle des boni homines ou des probi homines qui désigne ceux qui se voient reconnaitre par une communauté une certaine légitimité à la gouverner. En Languedoc, à défaut d'un consulat toujours onéreux et que rechigne parfois à payer une partie de la population, la majeure partie des villages de quelque importance se sont vus reconnaître la possibilité d'établir des syndics ou des procureurs. Le village de Saint-Guilhem-le-Désert, qui doit son origine à l'abbaye bénédictine de Gellone, possède ainsi trois syndics qui gèrent la communauté tout en reconnaissant l'autorité tutélaire de l'abbé. À l'échelle de la sénéchaussée de Carcassonne, il s'agit d'un lieu relativement notable puisqu'il compte une trentaine de feux à la fin du XIV $\mathrm{X}^{\mathrm{e}}$ siècle, ce qui correspond à environ 140 chefs de feu imposables, chiffre donné par une liste nominative établie en 1402. Le syndicat de Saint-Guilhem, à l'instar des autres institutions communales languedociennes, a tendance à être accaparé par une élite qui se réserve en particulier les postes de syndics; cette tendance est cependant à relativiser puisque sur la période 1380-1390, trente-cinq chefs de feu participent activement à la gestion de la communauté en occupant les fonctions de syndics, de capitaines, de leveurs de taille ou de notaires du syndicat. Environ un quart des chefs de feu imposables sont donc impliqués dans les affaires politiques et fiscales de la communautét ${ }^{46}$. Or, Saint-Guilhem est largement concerné par le Tuchinat: certains des villageois ont convergé vers Valmale pour y affronter, avec le

\footnotetext{
${ }^{46}$ C'est ce qui apparaît à travers le dépouillement des comptes conservés pour la communauté de Saint-Guilhem-le-Désert; Archives départementales de l'Hérault, 170 EDT, CC 29.
} 
soutien d'autres milices communales, les troupes du duc de Berry et les syndics accueillent dans leurs murs des Tuchins auxquels ils font donner à boire et à manger.

Autre exemple, celui de la ville de Bagnols-sur-Cèze dans la sénéchaussée de Beaucaire: Bagnols ne peut en aucun cas être considéré comme un village et s'est vu progressivement reconnaître le droit d'être représenté par quatre procureurs. De façon nettement plus marquée qu'à Saint-Guilhem, les fonctions de procureurs sont ici réservées aux membres d'une élite sociale aisément identifiable composée de marchands, de bourgeois et d'hommes de lois, même si une évolution qui tend à laisser un poste de procureur à un laboureur se dessine vers la fin du XIV ${ }^{\mathrm{e}}$ siècle $^{47}$. Or, même si elles s'en défendent par la suite, ces élites urbaines font preuve d'une étonnante passivité vis-à-vis des Tuchins, voire d'une certaine complicité. Les Tuchins de la région peuvent parader ouvertement sur la place de Bagnols, y circuler librement et venir y entreposer une partie de leur butin; en certaines occasions particulières telles que la récupération de bétail razzié par les gens d'armes, le capitaine de $\mathrm{Ba}$ gnols et ses hommes n'hésitent d'ailleurs pas à combattre aux côtés des Tuchins. Cette collaboration semble en partie la résultante d'une certaine pression sociale: lors d'une assemblée générale tenus dans le cloître de l'église paroissiale de Bagnols, les »menus « venus en masse auraient fait valoir que le métier de brassier auquel bon nombre d'entre eux se livraient nécessitait une protection armée et une sécurité des campagnes, possible seulement en cas d'accord avec les Tuchins. Ils auraient en outre menacé les procureurs de la ville de se livrer à un massacre généralisé de tous les riches si jamais ces derniers refusaient d'ouvrir la ville aux Tuchins. Sans doute aurait-on tort de s'en tenir à cette seule explication dans la mesure où l'argument de la contrainte est systématiquement avancé par les élites à l'issue d'une rébellion dans le but d'obtenir une grâce royale; l'idée d'une pression résultant de tensions sociales au sein des communautés n'en doit pas moins être retenue, d'autant plus que villes et campagnes ont été secouées à partir des années 1330 par de graves crises qui remettent en question l'unité des communautés ${ }^{48}$, crises qui se renouvellent et s'exacerbent en $1379-1380^{49}$. L'engagement des élites au sein des mouvements paysans s'avère être un moyen fictif de recréer cette unité

\footnotetext{
${ }^{47}$ Sur les institutions de Bagnols-sur-Cèze, voir Pierre BERAud, Histoire de la ville de Bagnols-sur-Cèze, Nîmes 1941 .

${ }_{48}$ Sur ces crises dans les campagnes languedociennes, voir BOURIN-DERRUAU, Villages médiévaux (voir n. 26), vol. 2, p. 321-330. En ce qui concerne les tensions sociales au sein des villes, voir Philippe WOLFF, Les luttes sociales dans les villes du Midi français dans: Annales E. S. C. (1947), p. 443-454.

${ }^{49}$ On notera en particulier la conjonction d'émeutes anti-fiscales comme celles du Puy-enVelay et de Montpellier en 1378 et 1379 et de mouvements dirigés contre l'oligarchie urbaine comme celui de Béziers en 1381.
} 
menacée et peut s'analyser comme une tentative de prévenir les débordements populaires.

Plus fondamentalement peut-être, les élites rurales et urbaines sont, de part leur position politique, les interlocuteurs privilégiés du pouvoir royal et sans doute les seuls dont il dispose. La sortie de crise, pour une monarchie qui n'a rien à gagner à massacrer ses propres paysans, passe nécessairement par l'établissement d'un dialogue avec les révoltés ou leurs représentants. Pour dire les choses de manière un peu cynique, il me semble que les agents du roi ont tout intérêt à ce que les élites maintiennent le contact avec les rebelles de manière à pouvoir les utiliser par la suite lors des tractations de paix. D'ailleurs, si l'engagement de ces élites au sein d'une révolte représente toujours un risque, il est également pour elles une nécessité afin d'éviter de perdre tout poids politique et toute représentativité au sein d'une communauté donnée. Seule cette représentativité leur permet ensuite de se poser en garant du "bien commun« vis-à-vis des autres villageois et de faire accepter par les révoltés le retour à l'ordre. Ce processus est notamment à l'œuvre dans le cas du Tuchinat languedocien où le conseil du duc de Berry passe par l'intermédiaire de ces élites pour négocier avec les capitaines tuchins et pour faire accepter par la population l'énorme amende de 800000 francs infligée par la monarchie. Le maintien de la position politique des élites leur impose donc d'être solidaires des révoltes de type communautaire; à l'inverse, la monarchie, faute de pouvoir traiter directement avec les rebelles, a besoin que ces élites aient été impliquées dans ces révoltes pour négocier par leur biais. Ceci expliquerait la relative clémence de la monarchie envers ces élites à l'issue des révoltes paysannes, laquelle contraste durement avec l'impitoyable répression qui s'abat en revanche sur les paysans: en Île-de-France, les gens de métier semblent surreprésentés dans le corpus des lettres de rémission par rapport à leurs poids réel dans la jacquerie; en Languedoc, ni les syndics de Saint-Guilhem, ni les procureurs de Bagnols ne sont poursuivis par la monarchie et certains hommes de loi comme Pierre Boyer poursuivent même des carrières spectaculaires. Mais dans l'un et l'autre cas, massacres et pendaisons sommaires se multiplient sur la personne des paysans sans d'ailleurs se limiter aux zones et aux moments de combat et ce constat peut être élargi à la répression anglaise de l'insurrection cauchoise de $1436^{50}$.

Les révoltes paysannes de la fin du Moyen Âge, si liées à l'insécurité des campagnes et à l'incapacité de la monarchie à y apporter une réponse satisfaisante, doivent être vues à la fois comme des mouvements d'autodéfense et comme des moyens d'engager une discussion de nature politique avec des autorités seigneuriales ou royales, discussions dont les élites sociales ne peuvent se permettre d'être écartées sous peine de voir remise en cause leur position

${ }^{50}$ BoIs, Crise du féodalisme (voir n. 43), p. 299-304. 
dominante. S'il ne faut pas renoncer à voir dans cet engagement un véritable choix qui peut résulter de stratégies ou de convictions personnelles, il convient également de l'analyser comme une volonté de se maintenir dans une position politique intermédiaire, position qui fait des snotablesı à la fois les représentants naturels de leur communauté et les interlocuteurs du pouvoir royal. $\mathrm{La}$ participation des élites sociales aux révoltes paysannes est ainsi, au-delà de la conjonction de facteurs économiques, la résultante d'un choix, d'un risque et d'une stratégie qui engage d'abord et avant tout le groupe social dominant. Il est en revanche difficile de discerner de véritables tactiques au niveau familial au sein des communautés rurales: l'ascension sociale par le biais de la révoite semble concerner au premier chef des élites urbaines à l'exemple de Pierre Boyer ou des nobles comme Pierre de Bré, malgré son échec final. Il reste que cet engagement massif des élites rurales dans les révoltes paysannes de la fin du Moyen Âge s'explique largement par leur caractère communautaire: l'unanimité dans la rébellion contraint les élites à y adhérer, totalement ou partiellement, sous peine de s'exclure de leur propre communauté. 